\title{
Robot development project with human- and eco-friendly smart materials
}

\author{
Yoshiaki Itoh ${ }^{1,2^{*}}$, Toru Yamazaki ${ }^{3}$, Hun-ok Lim ${ }^{3}$ and Kenichi Takemura ${ }^{3}$
}

\begin{abstract}
The social need for robotic technology is rapidly increasing to prepare for the aging society in Japan. Many political action plans concerning Japan's robot strategy have been proposed and carried out nationwide. This project focused on the social implementation of life support robots and developing corresponding robotic technology. The project's participants were mainly selected from four laboratories in the department of Mechanical Engineering of Kanagawa University. In addition to robotic technology, there was participation and collaboration in this project from several specialized fields of mechanical engineering, such as material properties, material development, and mechanical vibration. Developing academia-industry-government collaboration was another key project aim. Prior to this project, joint research on dynamics and design concerning mechanical vibration and noise has been undertaken since 2011 by Kanagawa University and Kanagawa Industrial Technology Center. Based on this experience in project-based research, we extended our collaborative research, and the joint research project "Robot development project with human- and eco-friendly smart materials" started in April 2012 and ended in March 2014. In this paper, we report the research activities and results for this collaboration within the regional research community. First, we introduce the project planning. We discuss the relationship between the main theme of robotic technology and the subthemes, determined by the expertise of the members. We considered the safety and reliability of robotic technology in addition to developing materials and robot mechanics. In particular, we decided to pursue participation from fields that do not always engage directly with robotic technology. Second, we outline how we determined the subthemes of the project and we examine the contribution of each subtheme to the results of the main project. Moreover, we discuss our future development work. Finally, as a first step to implementing robotic technology in the regional social system, we report a recognition survey about the practical use and materials of robots that was performed among small and medium-sized enterprises related to robotic technology in the Kanagawa area. Through the survey, we improved the development and direction of the project. Furthermore, the exhibition "Robot Innovation 2012" held in the Tokyo International Exhibition Center communicated our project results to the public.
\end{abstract}

Keywords: Academia-industry-government collaboration, Human- and eco-friendly, Smart, Social activities, Robot impact reduction system, Material properties, Mechanical vibration

\section{Background}

The social need for robotic technology is rapidly increasing to cope with the aging society in Japan. In particular, service robots, including life support robots, must coexist and collaborate with humans in a common space.

\footnotetext{
*Correspondence: itoh@kanagawa-iri.jp

${ }^{1}$ Mechanical and Material Technology Division, Kanagawa Industrial

Technology Center, 705-1 Shimo-Imaizumi, Ebina, Kanagawa 243-0435, Japan

Full list of author information is available at the end of the article
}

New challenges arise from the coexistence of robots with humans that have never been considered for industrial robots because safety barriers usually separate them from humans. However, situations in which service robots work with humans could pose risks for humans and result in serious accidents. For example, collisions between humans and robots, robots falling, and mechanical and electrical malfunction of robots could occur. These problems must be addressed during innovation and social implementation to ensure that robots are safe and reliable [1]. 
Many political action plans concerning Japan's Robot Strategy have been proposed and conducted by the ministry of economy, trade and industry (METI). Several national projects and consortiums concerning robotics have been established and coordinated by the National Institute of Advanced Industrial Science and Technology, and the New Energy and Industrial Technology Development Organization. Similarly, at a regional level, many activities have been performed to meet specific regional needs, where local governments, universities, and companies have collaborated. For example, in Kanagawa, the Kawasaki-Kanagawa Robot Business Conference was established in 2006 prior to nationwide commercialization and marketing of service robots, and the members were the Kanagawa Prefectural Government and Kawasaki City Government. Since 2010, the conference has been operated by volunteer committees [2].

Kanagawa University and the Kanagawa Industrial Technology Center, which is a public institute in the Kanagawa Prefectural Government for supporting small and medium-sized enterprises (SMEs), have collaborated on research on the dynamics and design concerning mechanical vibration and noise since 2011. The founder members were the authors, Professor Yamazaki, along with the Yamazaki laboratory members, and Visiting Professor Itoh. Through this research, a key goal was to achieve academia-industry-government collaboration.

Based on this experience and the procedure that was established for project-based research, we decided to extend our collaborative research. We proposed a joint research project to the academia-industry-government Liaison Department, Kanagawa University. The joint research project "Robot development project with human- and eco-friendly smart materials" started in April 2012 and ended in March 2014. The main purpose of this project was to implement life support robots and develop the corresponding robotic technology. The project members were mainly from four laboratories in the Department of Mechanical Engineering, Kanagawa University. In addition to robotic technology, there was collaboration from several specialized fields of mechanical engineering, such as material properties, material development, and mechanical vibration.

In this paper, we report the activities and results for this collaboration among the regional joint research community. First, we describe the project planning. The relationship between the main theme of robotic technology and its subthemes, determined by the expertise of the members, is discussed from the viewpoint of the robot safety and reliability, in addition to the development of materials and robotic mechanics. In particular, we decided to seek participation from fields that have not always engaged directly with robotic technology. Second, the procedure by which we determined the subthemes is shown along with main theme of the project. We examine the contribution of the results of each subtheme to the main project result, and we discuss the future development of the project. Finally, as a first step to implementing robotic technology in the regional social system, a recognition survey on the practical use and materials for robots was conducted among SMEs relevant to robotic technology in Kanagawa. Through the survey, we improved the development and study direction of the project by using the plan-do-check-adjust (PDCA) cycle. Furthermore, the exhibition "Robot Innovation 2012" held in the Tokyo International Exhibition Center communicated the results of our project to the public.

\section{Methods}

\section{Aim and vision of the project Background to the project planning}

The research support system "Joint Research Incentive Grants" is available in the academia-industry-government Liaison Department of Kanagawa University. The project, supported by the grants, involved collaboration between Kanagawa University and Kanagawa Industrial Technology Center. We offered the opportunity for SMEs in Kanagawa to participate in our project. The project results were used to communicate the project to industry and society and promote collaboration between them. When the project was launched in 2011, the coexistence and collaboration between humans and robots had not been investigated. In fact, the "Robot Devices for Nursing Care Project" was released in November 2012 by METI and the Ministry of Health, Labor and Welfare.

Our project was set up to help create a society where humans and robots coexist. Robot-related technologies are a key research area for meeting social needs and have already been studied by the project members. Moreover, the project members have already collaborated on research into peripheral technology. Prior to the project, there were several discussions on topics such as the safety, commercialization, and the wider social implications of robotic technology. The members were from four laboratories: Professor Lim, Professor Takemura, Professor Kudo (at that time), and Professor Yamazaki and their laboratory members. The author, Professor Itoh, was a member of the Yamazaki laboratory as visiting professor and a researcher at Kanagawa Industrial Technology Center. The project started in April 2012 and ended in March 2014.

\section{Policy and direction of the project}

The main theme of the project was "The Safety of Robotic Technology" based on the research of the Lim laboratory, which played an important technical role. The research 
seeds from each participating laboratory were used for collaboration and applications in line with the policy and direction of the project. In particular, our members adapted their research to encompass the coexistence of human and robots. As a result, our members shared the policies and vision of the project.

Through discussions, we agreed on common definitions for the terms "robot" and "safety". Moreover, each laboratory also listed research themes for social contributions with "human-friendly", "eco-friendly", and "smart" as keywords.

" "Human-friendly" means safe.

* "Eco-friendly" means ecologically sound, recyclable, natural materials.

* "Smart" means a friendly appearance in addition to high performance.

Based on these discussions, our project "Robot development project with human- and eco-friendly smart materials" was carried out.

\section{Subthemes of the laboratories}

The following researchers and laboratories (titles and members at the time are listed) participated in the project:

* Lim laboratory:

Members: Professor H. Lim, Special Assistant H. Masuta

Research field: humanoid robots, human-robot interactions, robot safety mechanisms

* Takemura laboratory:

Members: Professor K. Takemura, Special Assistant H. Katogi

Research field: natural fiber-reinforced plastics, green composites, carbon fiber-reinforced plastics

* Kudo laboratory

Members: Professor K. Kudo

Research field: high-strength recycled metals

* Yamazaki laboratory

Members: Professor T. Yamazaki, Visiting Professor E. Kojima, Visiting Professor Y. Itoh

Research field: dynamics and design, vibration, sound intensity

The project leader was Professor T. Yamazaki. The project began with a minimal number of group members. The research field of our project was not necessarily specific to robotics and mechatronics. Consequently, the subthemes in the project were determined in accordance with the research fields of each participating project member.

Subtheme 1: development of human-friendly robotic technology for coexistence and collaboration in a common space

Subtheme 2: development of high-strength natural materials for use and support in robotic technology

Subtheme 3: development of high-strength recycled metals for use and support in robotic technology

Subtheme 4: dynamic evaluation of mechanical properties of new materials developed in Subthemes 2 and 3

The subthemes of the project were investigated, fully taking into account the strengths of each research field. Regular meetings among the project members to review the research results were held frequently to allow the project to benefit from the different expertise and perspectives of the members. The contribution and future development of the project subthemes were discussed. We used a new approach to give useful feedback on the survey results on the project. We included the survey as a further subtheme in our project.

Subtheme 5: survey of SMEs in the Kanagawa area on robotic technology

We examined the recognition of service robots among SME managers and engineers to deepen mutual understanding with the aim of filling any gaps by matching seeds from academic research with the needs of industry. In parallel to this project, we established a local community-based collaboration system, which benefits from the resources of the Kanagawa Industrial Technology Center. Therefore, we aimed to spread the robotic technology to society from both soft- and hard-infrastructure support.

\section{Results}

Outline of research results for each subtheme Subtheme 1: development of human-friendly robotic technology for coexistence and collaboration in a common space

This subtheme focused on the core robotic technology of the project and it was investigated by the Lim laboratory. In particular, human safety in physical human-robot interactions was studied in the following areas.

(a) Design and development of a passive collision force suppression mechanism

(b) Development of a human-friendly robot with a suppression mechanism in its joints

The other subthemes were investigated assuming that their results would be implemented in the robotic technology proposed by the Lim laboratory. In the spaces where humans and robots cooperate or coexist, humans are always exposed to the risk of collision, robots 
falling over, and mechanical and electrical malfunctions in the robots. To improve human safety, robots should be designed differently. In this research, a passive collision force suppression mechanism was designed and developed to reduce collisions. If the control system of a robot breaks down owing to software faults or the loss of electrical power, the suppression mechanism must be able to reduce impact and contact force passively.

Figure 1 shows a schematic of a robot manipulator system with a passive collision force suppression system for securing human safety [3]. The collision suppression system consists of an air cushioning cover that absorbs impulsive force and a passive collision suppression mechanism that disconnects the joints of the robot. Under normal operation, the mechanism acts with the motor torque when the clutch gear and the two linear guides in each manipulator joint are connected. If a collision between a human or an object and the manipulator occurs, the passive collision force suppression system is activated. The collision force is absorbed by the air cushion cover attached to the exterior of the robot manipulator. However, if the collision force exceeds a certain critical level, the robot's joints are disconnected, meaning that human safety is assured.

Figure 2 shows the details of the passive collision force suppression mechanism developed in 2012. The mechanism was installed in the manipulator and the waist of a human-friendly robot. In the first trial, the dynamic behavior of the robot manipulator with the suppression mechanism was confirmed. However, when a small collision force was applied to the robot, its manipulator and waist did not operate properly because the collision suppression mechanism was too heavy. This outcome had been predicted in the design stage. In the second trial, we used human- and eco-friendly smart materials developed in the project for part of the robot frame, which reduced

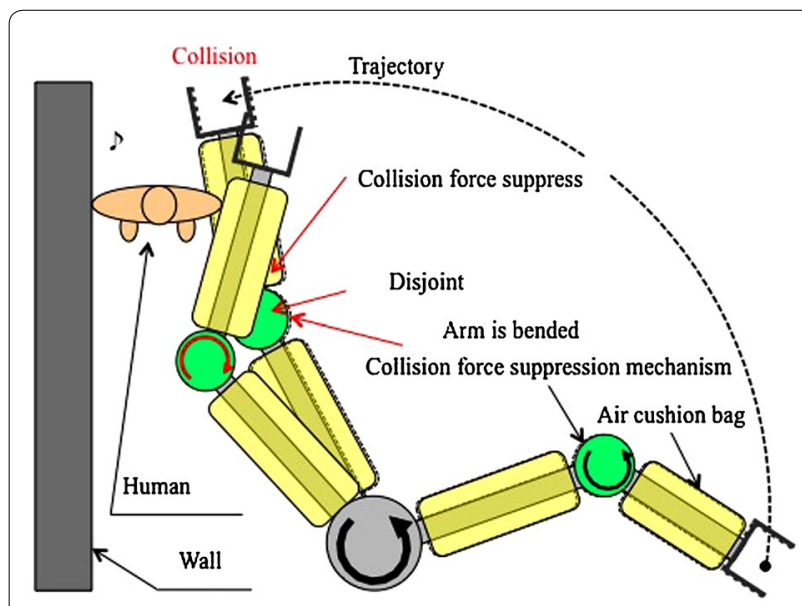

Fig. 1 Schematic of the robot impact reduction system for human safety

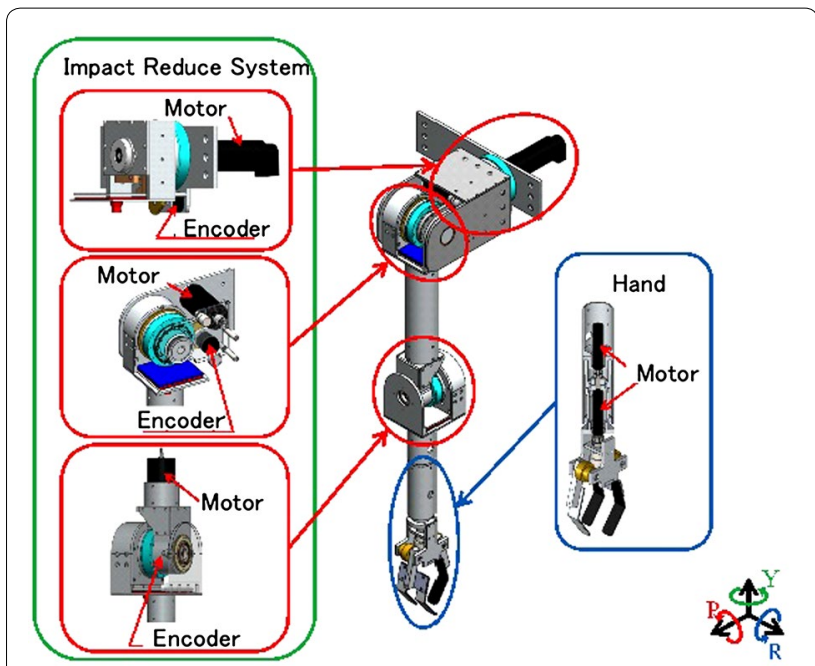

Fig. 2 Mechanism of the robot impact reduction system

the weight of the robot and improved the rigidity of its frame. We also changed the shape of some parts of the suppression mechanism and altered the material of the clutch teeth to improve their rigidity. Consequently, the collision mechanism operated correctly, improving reliability and human safety. Collision experiments between a human and a human-friendly robot were conducted that confirmed the effectiveness of the collision mechanism for ensuring human safety.

\section{Subtheme 2: development of high-strength natural materials for use and support in robotic technology}

This subtheme was investigated by the Takemura laboratory. This laboratory researches the strength of natural fiber-reinforced plastic, green composites, and carbon fiber-reinforced plastics for aircraft and spacecraft, motorcycles, and automobiles to develop strong, lightweight materials [4].

For this project, a study of high-strength natural materials for use in the passive collision force suppression mechanism for robot manipulators was performed. Our green composite materials consisting of natural fibers and biodegradable resin were not sufficiently strong for the ecofriendly materials required for the robotic technology in the project. Therefore, it was necessary to improve and evaluate the strength and stiffness material properties of the green composite materials for use in the proposed passive collision force suppression mechanism for robot manipulators. Applying the fibers to enforce the green composite as a surface treatment was expected to increase the strength of the material. Cellulose nanofibers (Fig. 3), which are an environmental friendly material made of wood pulp with high strength and stiffness, were used. Therefore, green 

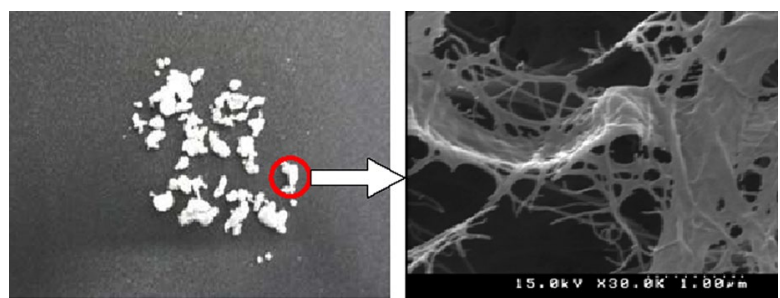

Fig. 3 Cellulose nanofibers

composites consisting of cellulose nanofiber ( 0,3 or $5 \%)$, natural fiber, and biodegradable resin for robot parts were fabricated and their flexural properties were examined.

The flexural properties of the green composite containing cellulose nanofibers are shown in Fig. 4. The flexural properties of the composite containing $3 \%$ nanofibers were higher than the composite containing no nanofibers. However, the flexural properties of the composite containing $5 \%$ nanofibers were lower than the composite containing 0 and $3 \%$ nanofibers. The optimum nanofiber content in green composites was $3 \%$, and this composite was used for robot parts.

\section{Subtheme 3: development of high-strength recycled metals for use and support in robotic technology}

This subtheme was investigated by the Kudo laboratory. This laboratory researches the strength of recycled stainless steels to develop new strong, lightweight materials, and focuses on heat treatments, such as quenching and annealing, to fabricate high-strength recycled metals. The heat treatment makes the crystal grain boundaries clearer, improving the damping characteristics [5]. The Yamazaki laboratory evaluated the dynamics of the

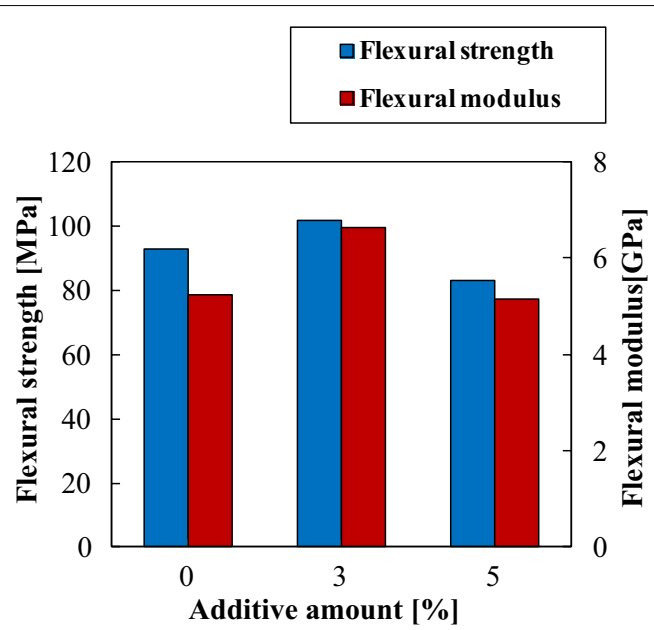

Fig. 4 Flexural properties of green composites containing cellulose nanofibers mechanical properties of the materials by combining analysis techniques for vibration energy propagation, and the Yamazaki laboratory showed the possibility that the materials were suitable for the robotic system.

\section{Subtheme 4: dynamic evaluation of mechanical properties of new materials developed in Subthemes 2 and 3}

This subtheme was investigated by the Yamazaki laboratory. This laboratory researches dynamics, design, mechanical vibration and noise, and sound intensity in areas such as automobiles, consumer electronics, and musical instruments to examine energy transfer in systems. Moreover, decreasing mechanical vibrations is a challenge for achieving higher quality life support. In particular, a time-frequency analysis method with digital filter developed in 2012 can be used for steady-state and time-dependent analyses of vibrations from the start of the vibration to steady-state conditions [6].

The dynamic mechanical properties of new materials were evaluated. Figure 5 shows the outline of the timefrequency analysis method. The input signal in Fig. 5a was analyzed by time-frequency analysis, which was expressed as the time-frequency distribution shown in Fig. 5b. Here, the horizontal and the vertical axes represent time and frequency, respectively, and the color bar represents the amplitude of the vibration. Moreover, we identified the damping coefficient of the new materials Fig. 5c. Time-frequency analysis was used to evaluate the green composite materials and the recycled metals.

Figure 6 shows the experimental setup and representative experimental results for the new materials. There was a tradeoff relationship between the strength and material damping; as the strength of the materials increased, the damping coefficient of the materials decreased. In the robots, the moving parts, such as motors or gears, were housed by the new materials. During operation, these moving parts become a sound source, and sound is transmitted to the external space and is audible by the user. To satisfy the criterion for "smart" materials, materials that damp vibration and transmit sound less should be developed. Thus, we examined the evaluation and modeling method for the acoustic transmission characteristics of a sound transmission prediction method. We investigated analytical and experimental statistical energy analysis (SEA) as the prediction method and modeling technique, and we confirmed the efficiency of the analysis in the high frequency range. The SEA method is also suitable for the development of aircraft, automobiles, and trains.

\section{Subtheme 5: survey of SMEs in the Kanagawa area on robotic technology}

As the social activity for promotion of interaction with local community, a recognition survey on the practical 


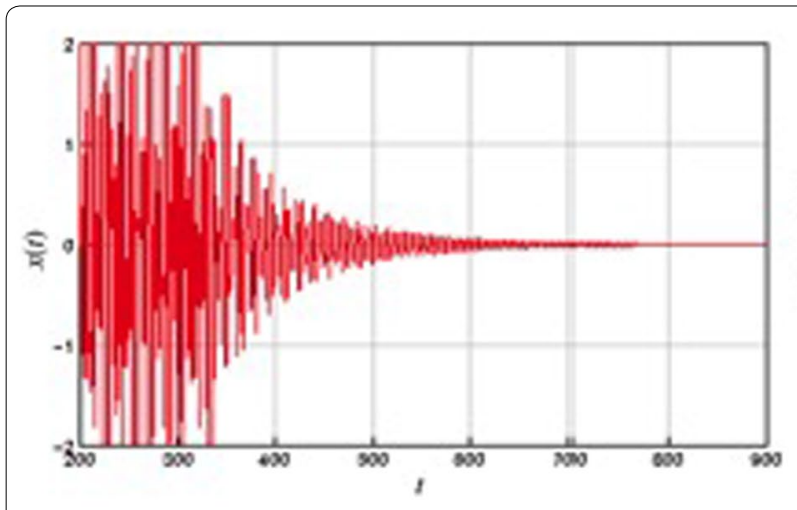

a

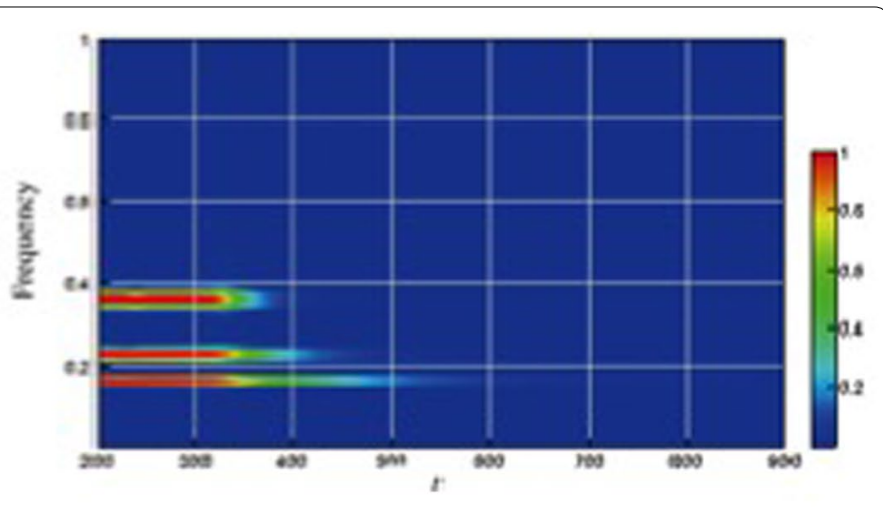

b

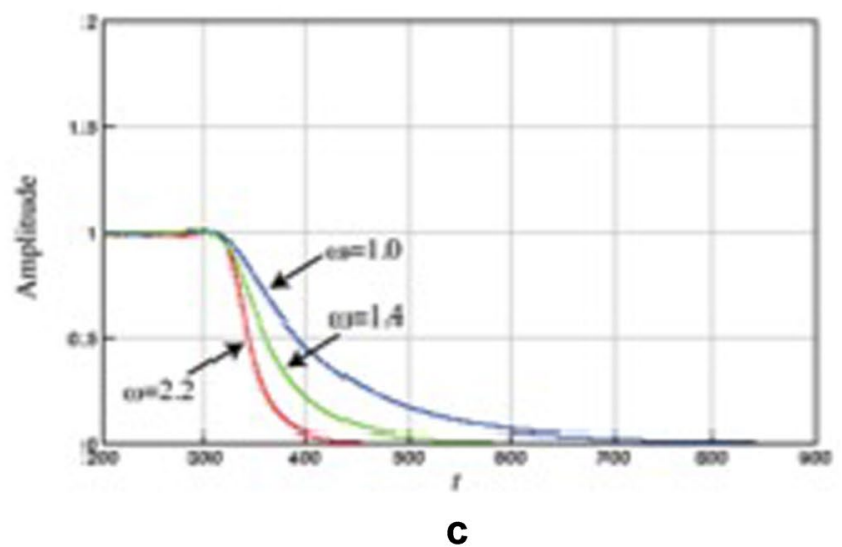

Fig. 5 Time-frequency analysis procedure $\mathbf{a}$ Input signal, $\mathbf{b}$ time-frequency analysis, $\mathbf{c}$ parameter identification of damping coefficient

use and material for the robot was carried out among SMEs involved in robotic technology in Kanagawa. We improved the development and direction of the project by using the PDCA cycle based on the feedback from the survey results. In particular, for the SME managers we visualized several scenarios in which the robots could be used. We also identified the research seeds and business needs at the same time. We constructed a community-based collaboration system that capitalized on the strength of the Kanagawa Industrial Technology Center. We aimed to spread the robotic technologies from both sides of soft- and hard-infrastructure support, so that the results of the project would be diffused to society to realize future robotic technology.

In the survey, we focused on nursing care where humans and robots coexist and co-work in the same space, as a typical example. In nursing care, prevention and relief of low back pain for care staff is important. The tasks that care workers dislike most involve transferring patients from one place to another, such as between beds and wheelchairs, because these tasks often cause low back pain. Safety in the co-working space was examined, and a survey about the anticipation and recognition of the new materials was also conducted.

In discussing the development and diffusion of robotic technology to an aging society, the terms "humanfriendly", "eco-friendly" and "smart" are important. In particular, the term "smart" was translated from the original Japanese word "Kibi" (literally, "subtlety") [7], though it is difficult to capture its nuance in English. The term conveys the sense of a nonliteral meaning that is subtle and has its origins in human emotions. Thus, the intended distinction is that "smart" refers not to "artificial intelligence" but rather to advanced capabilities as well as adaptability and applicability in tasks involving humans. This use of "smart" for robotic technology is new, and it could be misleading because the word can be interpreted differently. Therefore, we must promote the definition of "smart" as encompassing an intuitive interpretation. We also found that the spread of strategic terms is required. The survey for gauging social understanding of service robots and their peripheral technologies was crucial for the project. 

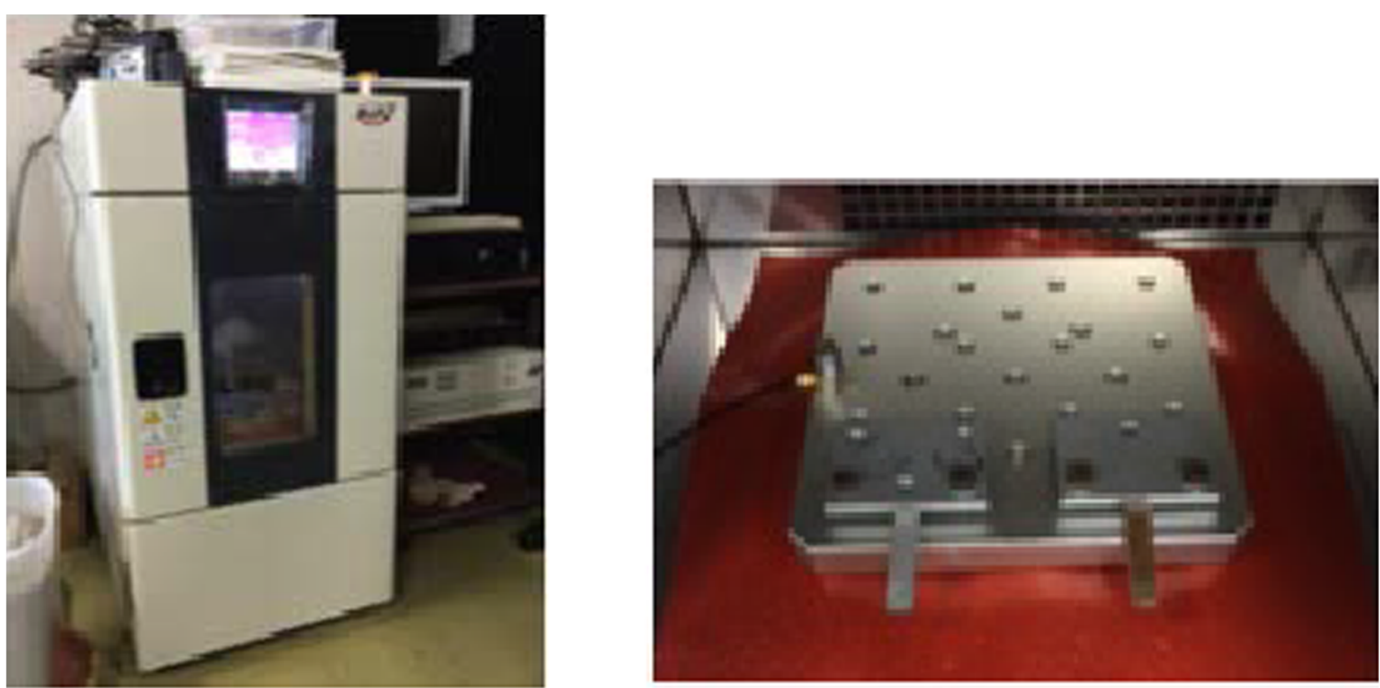

a

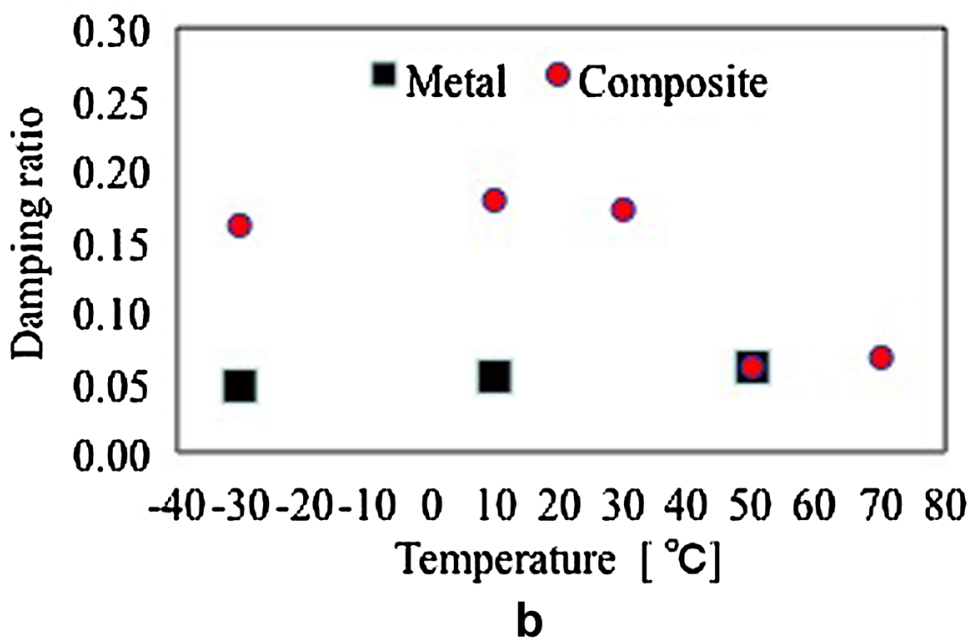

Fig. 6 Experimental results a experimental apparatus, b damping ratio

\section{Discussion}

\section{Communicating the project to industry and society}

The effectiveness of the passive collision force suppression mechanism for the robot manipulator developed in the project was verified by performing a variety of crash tests in the laboratory. The project results were communicated at the exhibition "Kanagawa Robot Innovation 2012" held in the Tokyo International Exhibition Center to promote our activities with a view toward future collaboration with industry. This exhibition was mainly organized by the Kanagawa Prefectural Government, and Professor Itoh was a core member of the exhibition committee [8]. The exhibition was part of "Japan Robot Week 2012" to promote service robots and their technology.

Regional SMEs from Kanagawa attended the exhibition to promote technologies and products related to life support robots and develop sales channels. In the exhibition, the presentation stage was prepared for promoting the exhibitors from the Kanagawa area Fig. 7a. We exhibited the robot manipulator Fig. $7 \mathrm{~b}$ and a panel explaining the robotic technology investigated in our project Fig. 7c. During the exhibition, there were close technical exchanges between our project and SMEs in the Kanagawa area. Moreover, a future collaboration framework was constructed, and secondary outcomes were also obtained. These activities were published as a business report by the Kawasaki City Industrial Promotion Foundation [9].

In our exhibition booth, the passive collision force suppression mechanism for the robot manipulator was displayed [8]. It is rare that the development of robotic technologies and academic research is promoted by 


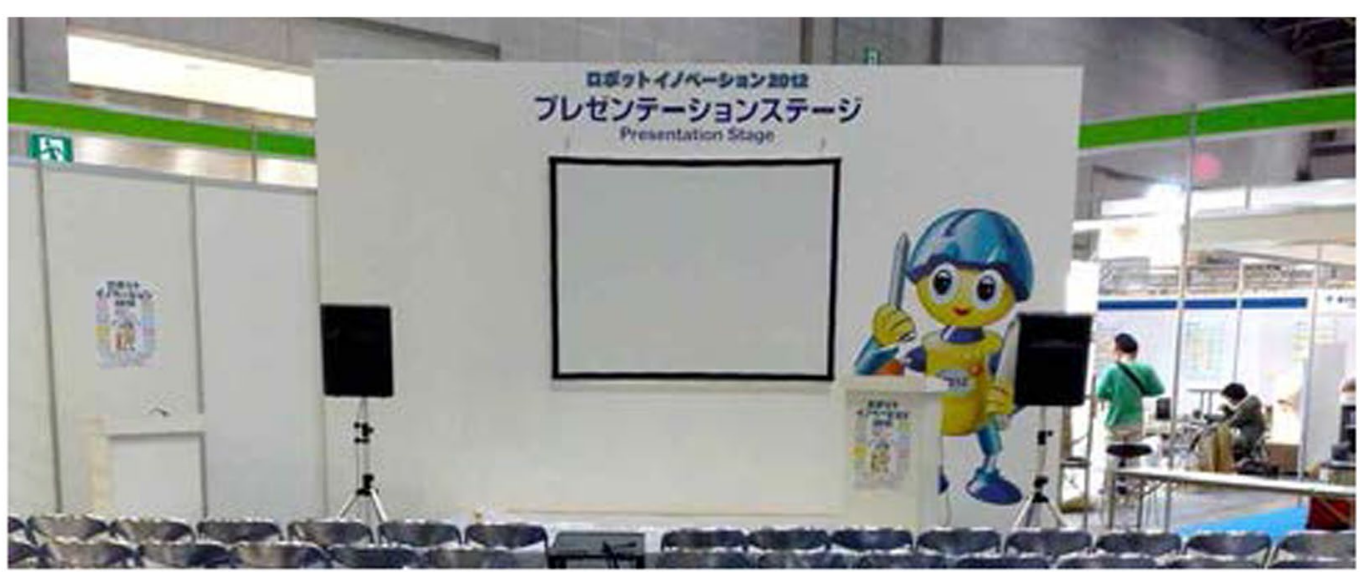

a

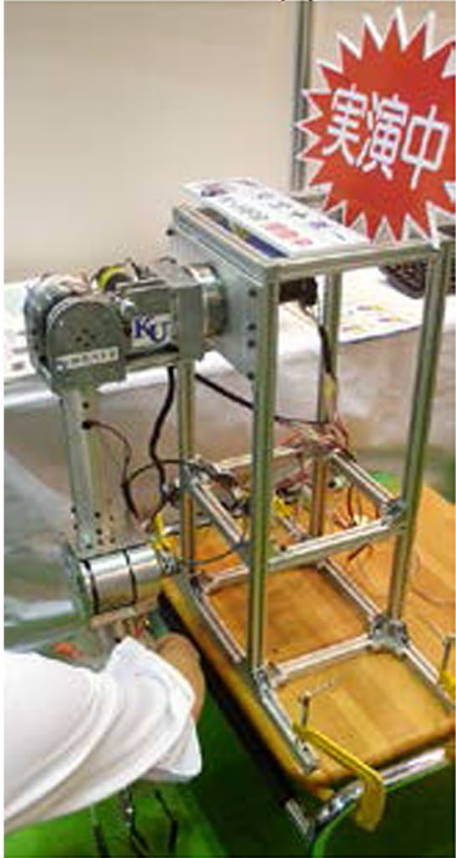

b

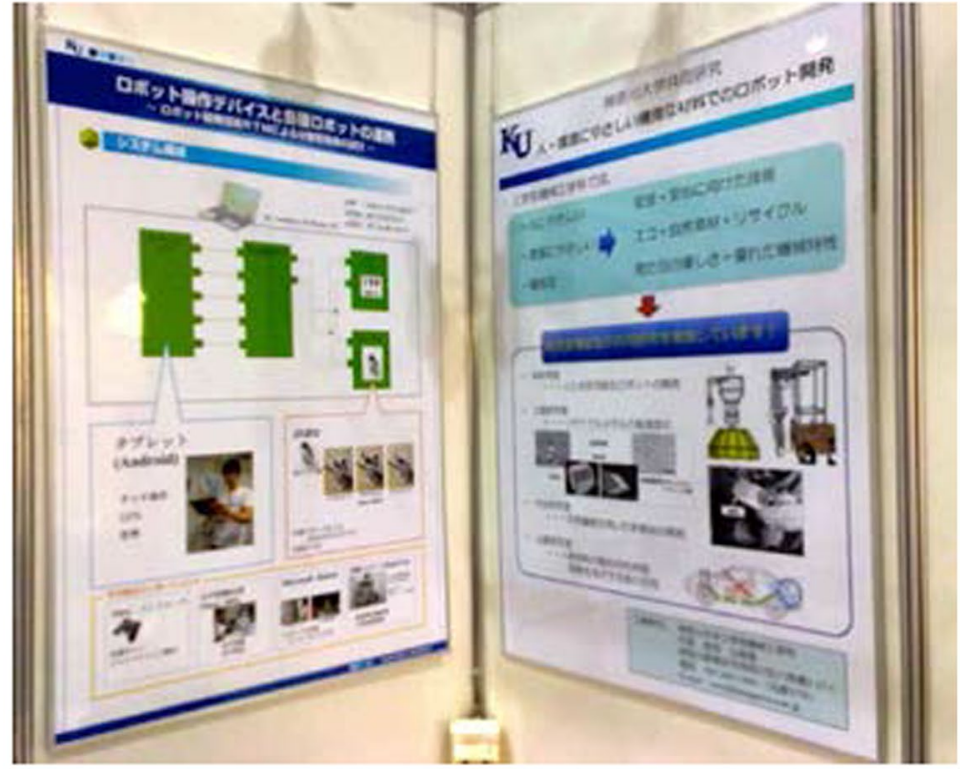

C

Fig. 7 Exhibition at Kanagawa Robot Innovation 2012 a presentation stage at Robot Innovation 2012 b robot [8] c panel

an initiative zone of the regional government in the industrial field. The collaboration between the SMEs and Kanagawa University was particularly successful, and can serve as a model case for industrial-academic collaboration.

\section{Conclusion}

The main purpose of the project was the development of life support robots and the corresponding robotic technology. Several fields of mechanical engineering besides robotic technology, such as material properties, material development, and mechanical vibration, collaborated in the project. In this paper, we reported the activities and results of the project for collaboration within the regional community. The collaboration extended over several laboratories to share expertise and different specializations, allowing us to complete the project and develop new research fields. We created a robot manipulator with a passive collision force suppression mechanism, and developed the robotic technology and new materials. We also performed the dynamic evaluation of the materials by time-frequency analysis. All this work proceeded on 
schedule. Through collaboration, the "Robot development project with human- and eco-friendly smart materials" project achieved its aims. Furthermore, for each subtheme, we achieved advancements in robotic technology, new material development, and mechanical vibration and noise. In mechanical engineering, these areas require further collaboration, and this will be addressed in future work.

Robotic technology and collaboration between research fields are early trends. Nevertheless, we have defined the concepts of "human-friendly", "eco-friendly", and "smart". Moreover, joint research based on the development of important robotic technology was performed to promote the project results within Japan and globally. This type of work is important for universities, and in future we would like to communicate our joint research results continuously.

\section{Authors' contributions}

TY led and directed the research, proposed the concept, designed the experiments, and submitted the proposal for ethical review. YI was the sub-leader and directed the development and exhibition management, drafted the manuscript, and analyzed experimental data with the time-frequency analysis method. HL designed and developed the passive collision force suppression mechanism for the robot manipulator. KT developed the high-strength natural materials for use and support in robotic technology. All authors read and approved the final manuscript.

\section{Author details}

${ }^{1}$ Mechanical and Material Technology Division, Kanagawa Industrial Technology Center, 705-1 Shimo-Imaizumi, Ebina, Kanagawa 243-0435, Japan. ${ }^{2}$ Research Institute for Engineering, Kanagawa University, 3-27-1 Rokkakubashi, Kanagawa-ku, Yokohama 221-8686, Japan. ${ }^{3}$ Department of Mechanical Engineering, Kanagawa University, 3-27-1 Rokkakubashi, Kanagawa-ku, Yokohama 221-8686, Japan.

\section{Authors' information}

YI received his B. Eng., M. Eng. and Dr. Eng. in mechanical engineering from Keio University, Japan in 1991, 1993, and 1996, respectively. From 1996 to 2007, he was a researcher at Kanagawa Industrial Technology Center (Kanagawa Industrial Technology Research Institute until 2005), Japan. From 2007 to 2011, he was an administrative officer in Kanagawa Prefectural Government. Since 2011, he has been a researcher at Kanagawa Industrial Technology Center, Japan. He has also been a part-time lecturer at Yokohama National University since 2011, and a visiting professor at Kanagawa University since 2012. His research interests are electromagnetics and dynamics, and nonlinear vibration in mechanics. He is a member of the Japan Society of Mechanical Engineers.

TY received his B. Eng. in mechanical engineering from Tokyo Institute of Technology, Japan in 1992, and then his M. Eng. in 1994 and Dr. Eng. in 1997 in mechanical engineering from Tokyo University, Japan. From 1998 to 2000, he was a lecturer at Kanagawa University, Japan, an associate professor from 2000 to 2010, and he has been a professor since 2010. He founded his company, Sentan Inc., Japan, in 2007 and serves as the company president. His research interests are mechanical vibration and noise from all kinds of sources, including musical instruments and the human body. He is a member of the Japan Society of Mechanical Engineers, the Society of Automotive Engineers of Japan, the Acoustical Society of Japan, the Institute of Noise Control Engineering of Japan, the Japan Fluid Power System Society, and the Japan Society of Civil Engineers, and he is a director of the Institute on Noise and Vibration Technology for Next Generation.

$\mathrm{HL}$ received his B. Eng. degree in the Department of Electrical Engineering from Hongik University, Korea, and then his M. Eng. and Ph.D. degrees in the Department of Mechanical Engineering from Waseda University, Japan. From 2000 to 2005, he was an associate professor in the Department of System Design Engineering, Kanagawa Institute of Technology. In April 2005, he joined the Department of Mechanical Engineering, Kanagawa University, where he is currently a Professor. Since 2000, he has also been with the Humanoid Robotics Institute, Waseda University, where he is currently a visiting professor. He has authored or coauthored more than 200 papers in international journals and conference proceedings. His research interests include humanoid robots, walking systems, mechatronics, human-robot symbiosis systems, image recognition, human safety control, and emotion-based control. He is an associate editor of the International Journal of Humanoid Robotics and is a Senior Member of IEEE.

KT received his B. Eng., M. Eng., and Dr. Eng. in mechanical engineering from Doshisha University, Japan in 1989, 1991, and 1994, respectively. From 1994 to 1997, he was a researcher at Dainippon Ink and Chemicals, Inc., Japan. From 1997 to 1999, he was a lecturer of Kanagawa University, Japan, and was an associate professor from 1999 to 2008, and has been a professor since 2008. $\mathrm{He}$ is a member of the Japan Society of Mechanical Engineers, the Society of Materials Science, Japan, and the Japan Society of Composite Materials.

\section{Acknowledgements}

This work was supported by "Joint Research Incentive Grants" in the academiaindustry-government Liaison Department of Kanagawa University.

\section{Competing interests}

The authors declare that they have no competing interests.

Received: 28 June 2016 Accepted: 1 September 2016 Published online: 13 September 2016

\section{References}

1. New Energy and Industrial Technology Development Organization (2013) Project for practical application of service robots. http://www.nedo.go.jp/ content/100388880.pdf

2. New Energy and Industrial Technology Development Organization (2014) White paper on robotization of industry, business and our life. http:// www.nedo.go.jp/content/100563893.pdf. Accessed 1 Sept 2016

3. Shimamoto K, Masuta H, Lim H (2012) Development of manipulator with collision force suppression mechanism. The 9th international conference on ubiquitous robots and ambient intelligence, pp 389-394

4. Takemura K, Miyamoto S, Katogi H (2013) Effect of surface treatment on creep property of jute fiber reinforced green composite under environmental temperature. Key Eng Mater 525-526:53-56

5. Akazawa T, Otake N, Kudo K (2001) Effect of recycling on the performance of air cellular cushioning materials. J Packag Sci Technol Jpn 10(6):289-295

6. Itoh Y, Yamaguchi N, Yamazaki T (2013) Vibration analysis based on timefrequency analysis with digital filter. J Syst Des Dyn 7-4:441-455

7. Itoh Y, Yamazaki T, Lim H, Takemura K (2015) Robot development project with human- and eco-friendly smart materials. Proceeding of the robotics and mechatronics conference 2015 in Kyoto, 1P2-D09 pdf

8. http://www.kanagawa-u.ac.jp/events/2012/09/17/004694/

9. http://www.kawasaki-net.ne.jp/sbk/event/report/jrw2012/index.html 